\title{
Semi-physiologically based pharmacokinetic modeling of paclitaxel metabolism and in silico- based study of the dynamic sensitivities in pathway kinetics
}

\author{
Martin N Fransson, Jan Brugard, Peter Aronsson and Henrik Green
}

\section{Linköping University Post Print}

N.B.: When citing this work, cite the original article.

riginal Publication:

Martin N Fransson, Jan Brugard, Peter Aronsson and Henrik Green, Semi-physiologically based pharmacokinetic modeling of paclitaxel metabolism and in silico-based study of the dynamic sensitivities in pathway kinetics, 2012, European Journal of Pharmaceutical Sciences, (47), 4, 759-767.

http://dx.doi.org/10.1016/j.ejps.2012.08.002

Copyright: Elsevier

http://www.elsevier.com/

Postprint available at: Linköping University Electronic Press

http://urn.kb.se/resolve?urn=urn:nbn:se:liu:diva-86649 


\title{
Semi-physiologically based pharmacokinetic modeling of paclitaxel metabolism and in silico-based study of the dynamic sensitivities in pathway kinetics
}

\author{
Martin N. Fransson ${ }^{\mathrm{a}, *}$, Jan Brugård $^{\mathrm{b}}$, Peter Aronsson ${ }^{\mathrm{b}}$, Henrik Gréen ${ }^{\mathrm{c}, \mathrm{d}}$ \\ ${ }^{a}$ Department of Medical Epidemiology and Biostatistics, Karolinska Institutet, \\ Stockholm, Sweden \\ ${ }^{b}$ MathCore Engineering AB, Linköping, Sweden \\ ${ }^{c}$ Division of Drug Research, Department of Medical and Health Sciences, Linköping \\ University, Linköping, Sweden \\ ${ }^{d}$ Science for Life Laboratory, School of Biotechnology, Division of Gene Technology, \\ KTH Royal Institute of Technology, Solna, Sweden
}

\begin{abstract}
Purpose: To build a semi-physiologically based pharmacokinetic model describing the uptake, metabolism and efflux of paclitaxel and its metabolites and investigate the effect of hypothetical genetic polymorphisms causing reduced uptake, metabolism or efflux in the pathway by model simulation and sensitivity analysis.

Methods: A previously described intracellular pharmacokinetic model was used as a starting point for model development. Kinetics for metabolism, transport, binding and systemic and output compartments were added to mimic a physiological model with hepatic elimination. Model parameters were calibrated using constraints postulated as ratios of concentrations and amounts of metabolites and drug in the systemic plasma and output compartments. The sensitivity in kinetic parameters was tested using dynamic sensitivity analysis.

Results: Predicted plasma concentrations of drug and metabolites were in the range of what has been observed in clinical studies. Given the final model,
\end{abstract}

\footnotetext{
${ }^{*}$ Corresponding author. Present address: Department of Medical Epidemiology and Biostatistics, Karolinska Institutet, PO Box 281, SE-171 77, Stockholm, Sweden. Tel.: +468524839 74; fax: +468314975.

Email address: Martin.Fransson@ki.se (Martin N. Fransson)
}

June 27, 2012 
plasma concentrations of paclitaxel seems to be relatively little affected by changes in metabolism or transport, while its main metabolite may be largely affected even by small changes. If metabolites prove to be clinically relevant, genetic polymorphisms may play an important role for individualizing paclitaxel treatment.

Keywords: Paclitaxel metabolism, Mathematical modeling, Pharmacokinetics, Sensitivity analysis, CYP2C8, CYP3A4, OATP, ABCB1

\section{Introduction}

Typically, parametric pharmacokinetic modeling starts with fitting oneor several compartments to observed drug concentrations in plasma from repeated sampling from one or several individuals. The latter case is subject to so called population pharmacokinetics, where the use of nonlinear mixed effects models allow variability in the population to be tested against potential covariates, such as body-mass, gender or genetic polymorphisms. Finding significant covariates is especially important for chemotherapeutic drugs because of their potency and narrow therapeutic index (Undevia et al., 2005).

In the case of paclitaxel, a mitotic inhibitor used in treatment for a range of different tumors, the use of population pharmacokinetic models has indicated sex, age, body weight and bilirubin to be significant covariates (Henningsson et al., 2003; Joerger et al., 2006). However, these covariates explain only a smaller part of the population variability, and it is believed that pharmacogenetics could play an important role in further reducing variability (Sparreboom and Figg, 2006). For paclitaxel, potential genetic polymorphisms affecting pharmacokinetics may be associated with the organic aniontransporting polypeptide (OATP) at cellular uptake (Smith et al., 2005), cytochromes P450 2C8 and 3A4/5 (CYP2C8, CYP3A4/5) (Monsarrat et al., 1993; Cresteil et al., 1994; Harris et al., 1994b; Rahman et al., 1994) during hepatic metabolism or the transporter protein ABCB1 at cellular efflux (Jang et al., 2001). Two large studies have so far been investigating genetic polymorphisms in association to paclitaxel pharmacokinetics, using the population pharmacokinetic approach (Henningsson et al., 2005a; Bergmann et al., 2011). While Henningsson et al. (2005a) found no associations for polymorphisms in CYP2C8, CYP3A4/5 or ABCB1, Bergmann et al. (2011) reported the allele $\mathrm{CYP} 2 \mathrm{C} 8 * 3$ to cause an $11 \%$ decrease in paclitaxel clearance. The same study also found evidence that the CYP2C $8 * 4$ and $\mathrm{ABCC} 1$ 
may have an effect on clearance, but found no significant influence of genetic variants in OATP, CYP3A4/5 or ABCB1. In contrast, a few minor studies have reported ABCB1 polymorphisms to affect either the kinetics of paclitaxel (Yamaguchi et al., 2006; Green et al., 2009) or of its hydroxy metabolites (Nakajima et al., 2005; Fransson et al., 2011).

In addition to the top-down pharmacokinetic modeling approach, where the study data may be a limiting factor for model complexity (Aarons, 2005), a complementary mechanistic approach may help to understand the relative importance of the targeted genes, and consequently what findings that may be expected from a clinical study. For this reason, the aim of the present work was to develop a semi-physiologically based pharmacokinetic model with detailed description of the uptake, metabolism and efflux of paclitaxel and its metabolites, by extending an existing in vitro derived intracellular pharmacokinetic model and integration of existing population pharmacokinetic models. The hypothetical effect of genetic polymorphisms causing reduced uptake, metabolism or efflux in the pathway was then investigated by the use of simulation-based dynamic sensitivity analysis.

\section{Material and methods}

\subsection{Model development}

A semi-physiologically based pharmacokinetic model was developed using the software MathModelica ver. 2.1 (MathCore Engineering AB, Linköping, Sweden), and combined low- and high-level pharmacokinetic models. An intracellular pharmacokinetic model used to assess the uptake and efflux of paclitaxel in tumor cells by Kuh et al. (2000) and Jang et al. (2001), was used as a starting point for model development. Pharmacodynamic effects on the number of cells were omitted. Kinetic parameters depending on cell number were up-scaled to represent a female liver of $1475 \mathrm{~g}$ (de la Grandmaison et al., 2001) with a hepatocellularity of $9.9 \times 10^{7}$ cells/g (Barter et al., 2007) resulting in a cell number of $1.5 \times 10^{11}$. The original model, comprising one compartment each for cells, considered here to be liver tissue, equation (3e), and cell medium, considered to be liver plasma, (3c), was then extended with a reservoir or systemic plasma compartment, (3a), and output compartment, (3g), to conceptually mimic a physiological model for hepatic elimination (Sirianni and Pang, 1997). A saturable transport mechanism, representing hepatic uptake facilitated by OATP (Smith et al., 2005), was introduced 
between the liver plasma compartment, (3c), and liver tissue compartment, (3e).

In hepatocytes, paclitaxel is mainly metabolized by CYP2C8 to $6 \alpha-$ hydroxypaclitaxel (Harris et al., 1994a; Kumar et al., 1994; Rahman et al., 1994), or by CYP3A4 to p-3'-hydroxypaclitaxel (Harris et al., 1994b). Both hydroxy metabolites can be further metabolized to $6 \alpha-$, p-3'-dihydroxypaclitaxel, by CYP3A4 or CYP2C8 (Sonnichsen et al., 1995). Hence, two metabolizing mechanisms, representing CYP2C8 and CYP3A4, were added in the liver tissue compartment, (3e), using Michaelis-Menten kinetics.

Because of its lipophilic properties, paclitaxel is usually administrated as an infusion, dissolved in the formulation vehicle Cremophor EL, which has been shown to affect the kinetics of the drug (Sparreboom et al., 1996), and most likely the kinetics of the two primary hydroxy metabolites (Fransson et al., 2011). Binding of drug to Cremophor EL and proteins in the systemic and liver plasma compartments, (3a) and (3c), was assumed to be instantaneous by including an equation derived from population pharmacokinetic modeling by Henningsson et al. (2001), describing the relation between total and unbound concentrations, (3b) and (3d). Cremophor EL concentrations were simulated using a previously reported three-compartment model (Henningsson et al., 2005b), also in the Appendix, equation A.5.

For each physiological compartment, four ordinary differential equations had to be used, each one representing the drug or one of the three metabolites. In total, the model consisted of a system of 19 ordinary differential equations, where the corresponding time-dependent variables are presented in Table 1. Initial estimates for physiological and kinetic parameters for paclitaxel were derived from various literature sources according to Table A.4. Because little kinetic data is available about paclitaxel metabolites, it was assumed that all binding parameters for metabolites are the same as for paclitaxel, with the exception of binding to Cremophor EL (Fransson et al., 2011). Initial values (pre-optimization) for enzyme kinetics of metabolites were also taken to be the same as the corresponding ones for the parent drug.

\subsection{Constrained optimization}

To mimic the in vivo situation as far as possible the semi-physiologically based model was subject to constrained optimization. Two time-points were chosen to constrain the model in such a way that the simulations would give reasonable ratios between metabolite and parent drug concentrations and amounts. To describe the relation between total concentrations of parent 
Table 1: Time-dependent variables

\begin{tabular}{ll}
\hline Variable & Meaning \\
\hline$x_{1}(t)$ & Total conc. $(\mu \mathrm{M})$ paclitaxel in systemic plasma \\
$x_{2}(t)$ & Total conc. $(\mu \mathrm{M})$ paclitaxel in liver plasma \\
$x_{3}(t)$ & Total conc. $(\mu \mathrm{M})$ paclitaxel in liver tissue \\
$x_{4}(t)$ & Total conc. $(\mu \mathrm{M}) 6 \alpha$-hydroxypaclitaxel in systemic plasma \\
$x_{5}(t)$ & Total conc. $(\mu \mathrm{M}) 6 \alpha$-hydroxypaclitaxel in liver plasma \\
$x_{6}(t)$ & Total conc. $(\mu \mathrm{M}) 6 \alpha$-hydroxypaclitaxel in liver tissue \\
$x_{7}(t)$ & Total conc. $(\mu \mathrm{M})$ p-3'-hydroxypaclitaxel in systemic plasma \\
$x_{8}(t)$ & Total conc. $(\mu \mathrm{M})$ p-3'-hydroxypaclitaxel in liver plasma \\
$x_{9}(t)$ & Total conc. $(\mu \mathrm{M})$ p-3'-hydroxypaclitaxel in liver tissue \\
$x_{10}(t)$ & Total conc. $(\mu \mathrm{M}) 6 \alpha-$, p-3'-dihydroxypaclitaxel in systemic plasma \\
$x_{11}(t)$ & Total conc. $(\mu \mathrm{M}) 6 \alpha-$, p-3'-dihydroxypaclitaxel in liver plasma \\
$x_{12}(t)$ & Total conc. $(\mu \mathrm{M}) 6 \alpha-$, p-3'-dihydroxypaclitaxel in liver tissue \\
$x_{13}(t)$ & Amount $(\mu \mathrm{mol})$ paclitaxel in output compartment \\
$x_{14}(t)$ & Amount $(\mu \mathrm{mol}) 6 \alpha$-hydroxypaclitaxel in output compartment \\
$x_{15}(t)$ & Amount $(\mu \mathrm{mol})$ p-3'-hydroxypaclitaxel in output compartment \\
$x_{16}(t)$ & Amount $(\mu \mathrm{mol}) 6 \alpha-$, p-3'-dihydroxypaclitaxel in output compartment \\
$x_{17}(t)$ & Conc. (ml/l) Cremophor EL in central compartment \\
$x_{18}(t)$ & Conc. $(\mathrm{ml} / \mathrm{l}) \mathrm{Cremophor} \mathrm{EL} \mathrm{in} \mathrm{first} \mathrm{peripheral} \mathrm{compartment}$ \\
$x_{19}(t)$ & Conc. (ml/l) Cremophor EL in second peripheral compartment \\
\hline
\end{tabular}

drug and metabolites in systemic plasma, an earlier developed model was simulated (Fransson et al., 2011), and the total concentrations of the population means at time $(t)$ at three hours, end of infusion, was noted. Total paclitaxel concentration $\left(x_{1}(t)\right)$ was taken as the reference level, and total concentrations of $6 \alpha$-hydroxypaclitaxel $\left(x_{4}(t)\right)$, p-3'-hydroxypaclitaxel $\left(x_{7}(t)\right)$ and $6 \alpha-$, p-3'-dihydroxypaclitaxel $\left(x_{10}(t)\right)$ was used to determine an appropriate ratio for total concentrations. Ratios of the amounts of $6 \alpha$-hydroxypaclitaxel $\left(x_{14}(t)\right), \mathrm{p}-3$ '-hydroxypaclitaxel $\left(x_{15}(t)\right)$ and $6 \alpha-, \mathrm{p}-3$ '-dihydroxypaclitaxel $\left(x_{16}(t)\right)$ to parent drug $\left(x_{13}(t)\right)$ in output were estimated using information about the mean extractable radioactivity from fecal collections as reported by Walle et al. (1995). Only the parent drug and three mentioned metabolites were considered, and the ratios were calculated from the sum of these four compounds. The constraint in the output compartment was set to $t=18$ hours, a compromise between amounts being stable (flattened curves) and 
computation time. Moreover, all the constraints were somewhat relaxed by allowing a $20 \%$ deviation from the reference level. The constraints are summarized in Table 2. Initial values for the model are presented in the Ap-

Table 2: Optimization constraints in systemic plasma and output compartment

\begin{tabular}{lll}
\hline Variable & Reference level & Constraint with $20 \%$ deviation \\
\hline$x_{4}(t)$ & $0.066 x_{1}(t=3)$ & $0.053 x_{1}(3)<x_{4}(3)<0.079 x_{1}(3)$ \\
$x_{7}(t)$ & $0.018 x_{1}(t=3)$ & $0.014 x_{1}(3)<x_{7}(3)<0.022 x_{1}(3)$ \\
$x_{10}(t)$ & $0.018 x_{1}(t=3)$ & $0.014 x_{1}(3)<x_{10}(3)<0.022 x_{1}(3)$ \\
$x_{14}(t)$ & $5.2 x_{13}(t=18)$ & $4.1 x_{13}(18)<x_{14}(18)<6.2 x_{13}(18)$ \\
$x_{15}(t)$ & $0.38 x_{13}(t=18)$ & $0.30 x_{13}(18)<x_{15}(18)<0.46 x_{13}(18)$ \\
$x_{16}(t)$ & $1.2 x_{13}(t=18)$ & $0.94 x_{13}(18)<x_{16}(18)<1.4 x_{13}(18)$ \\
\hline
\end{tabular}

pendix in Table A.4 and Table A.5. A manual optimization step using the MathModelica Simulation Center was first carried out to put the constrained variables approximately within a $50 \%$ deviation from the reference level. In this step, only $V_{\max }$ parameters where adjusted. Constrained optimization was then carried out in Mathematica ver. 8 (Wolfram Research, Inc., Champaign, IL, USA) using the NDSolve and FindMinimum routines, with several subsequent steps until all constraints were fulfilled with $20 \%$ maximum deviation from the reference level. In this second step, all $V_{\max }$ and related $K_{M}$ parameters, as well as the parameters describing binding to Cremophor EL, $B_{C r E L}$, were subject to the optimization procedure.

\subsection{Dynamic sensitivity analysis}

Dynamic sensitivity analysis was performed in MathModelica Simulation Center using the CVODES solver, which supports forward sensitivity analysis (Hindmarsh et al., 2005). The sensitivity $s_{i}$ for a parameter $p$ is calculated as

$$
s_{i, p}=\frac{\partial x_{i}}{\partial p}
$$

where $x_{i}$ is the $i$ th (state) variable. The forward sensitivity analysis will provide the local sensitivity for the parameters under consideration (Varma et al., 1999; Wu et al., 2008). This means that the results from the analysis are conditional on the specific parameter estimates, and that the sensitivity may not be valid for another set of estimates. 
Sensitivity was tested on all $V_{\max }$ and related $K_{m}$ parameters by first introducing a parameter, GE ("genetic effect"). This parameter effects either the $V_{\max }$ or the $K_{m}$ independently of the substrate. For instance

$$
V_{\max O A T P}^{\text {pac }} \rightarrow G E_{O A T P}^{V_{\max }} \cdot V_{\max O A T P}^{\text {pac }}, G E_{O A T P}^{V_{\max }}=1
$$

so that the sensitivity in the parameter $G E_{O A T P}^{V_{\max }}$ can be tested. This way, the sensitivity will be independent of the magnitude of the different $V_{\max }$, and the effect of reduced capacity in transporters and enzymes can be compared.

\section{Results}

\subsection{Model development and constrained optimization}

The final model for paclitaxel is described by equations (3a)-(3g), where the differential equations (3a), (3c) and (3e) represent the kinetics for total concentrations of paclitaxel, $x_{1}-x_{3}$, and equations $(3 \mathrm{~b}),(3 \mathrm{~d})$ and $(3 \mathrm{f})$ represent the relation between total, $x_{1}-x_{3}$, and unbound concentrations, $y_{1}-y_{3}$. Equation $(3 \mathrm{~g})$ represents the change in amount of drug, $x_{13}$, in the output compartment. The descriptions of each variable can be found in Table 1. The full model including kinetics for metabolites is represented by Figure 1, and all the underlying equations can be found in Appendix A.

$$
\begin{aligned}
& V_{\text {SysPl }} \cdot \dot{x}_{1}=-Q_{\text {LivPl }}\left(x_{1}-x_{2}\right)+\text { Dose }^{p a c} \\
& x_{1}=y_{1}+\left(B_{l i n P l}+B_{C r E L}^{p a c} \cdot x_{17}\right) \cdot y_{1}+\frac{B_{\max P l} \cdot y_{1}}{K_{m P l}+y_{1}} \\
& V_{L i v P l} \cdot \dot{x}_{2}=Q_{L i v P l}\left(x_{1}-x_{2}\right)-Q_{L i v T i}\left(y_{2}-y_{3}\right)-\frac{V_{\operatorname{maxOATP}}^{p a c} \cdot y_{2}}{K_{m O A T P}^{p a c}+y_{2}} \\
& x_{2}=y_{2}+\left(B_{l i n P l}+B_{C r E L}^{p a c} \cdot x_{17}\right) \cdot y_{2}+\frac{B_{\max P l} \cdot y_{2}}{K_{m P l}+y_{2}} \\
& V_{L i v T i} \cdot \dot{x}_{3}=Q_{L i v T i}\left(y_{2}-y_{3}\right)+\frac{V_{\operatorname{maxOATP}}^{\text {pac }} \cdot y_{2}}{K_{m O A T P}^{\text {pac }}+y_{2}} \\
& -\frac{V_{m a x 2 C 8}^{p a c} \cdot y_{3}}{K_{m 2 C 8}^{p a c}+y_{3}}-\frac{V_{m a x}^{p a A 4} \cdot y_{3}}{K_{m 3 A 4}^{p a c}+y_{3}}-\frac{V_{m a x A B C}^{p a c} \cdot y_{3}}{K_{m A B C}^{p a c}+y_{3}} \\
& x_{3}=y_{3}+B_{l i n T i} \cdot y_{3}+\frac{B_{\operatorname{maxTi}} \cdot y_{3}}{K_{m T i}+y_{3}} \\
& \dot{x}_{13}=\frac{V_{\max A B C}^{\text {pac }} \cdot y_{3}}{K_{m A B C}^{\text {pac }}+y_{3}}
\end{aligned}
$$




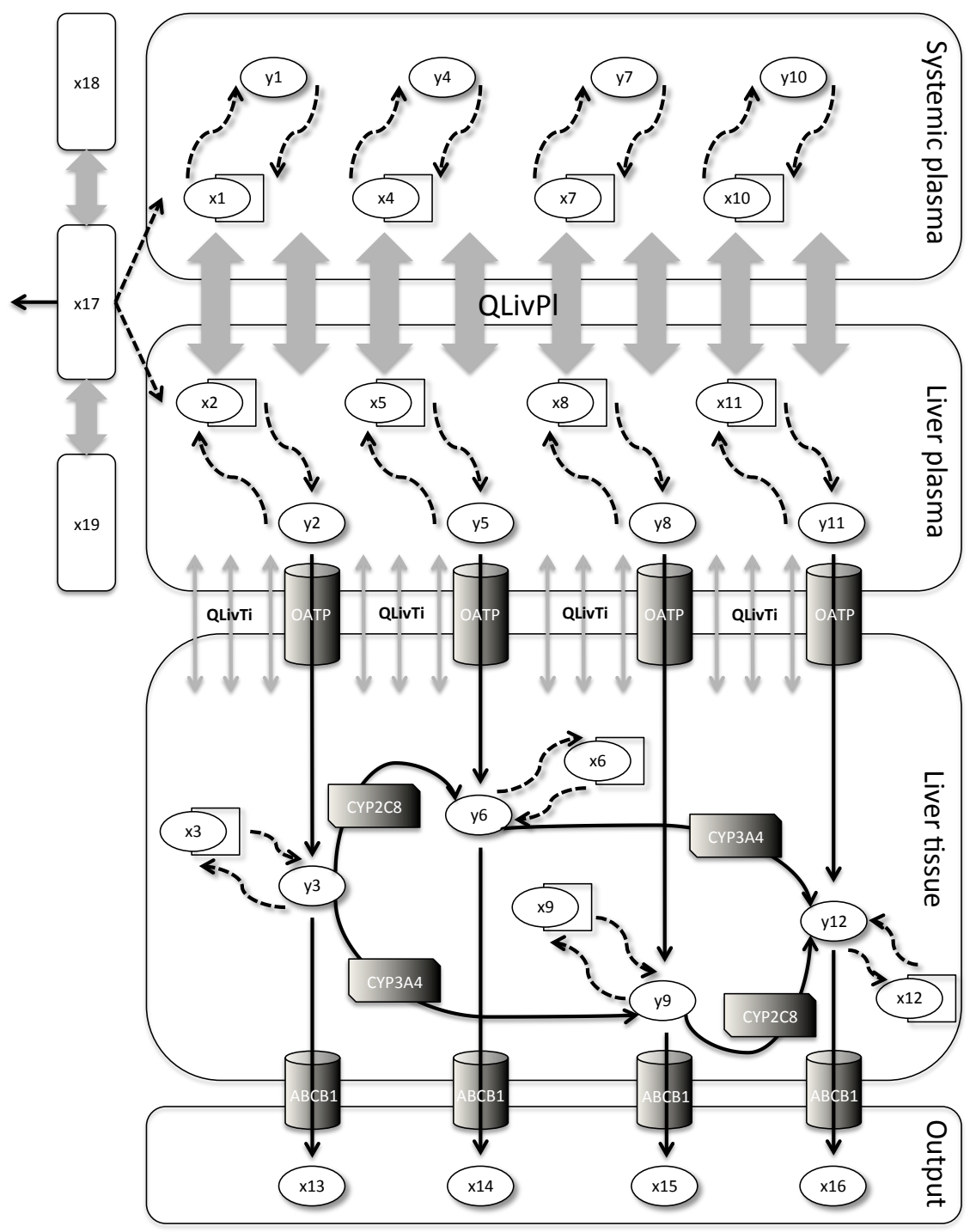

Figure 1: The final model structure. $x_{1}-x_{19}$, time-dependent variables according to Table 1 and $y_{1}-y_{12}$, the corresponding unbound concentrations. Black dashed arrows represent binding, black solid arrows represent enzyme kinetics and double-edged gray arrows represent compartmental flows. 
The parameter estimates from the constrained optimization is presented in Appendix A, Table A.5. The concentration of total paclitaxel and metabolites in the systemic plasma, liver plasma, liver tissue compartments, as well as the amount in the output compartment from a 20 hour simulation of the final model with a three-hour infusion is shown in Figure 2.

The final step during the optimization consisted of taking the estimates from a $30 \%$ to a $20 \%$ maximum deviation. Using the Mathematica Timing function, the last step was measured to approximately 6.1 hours of computational time on a Lenovo T61 with Intel Core Duo CPU $2.00 \mathrm{GHz}$ and 1.96 GB RAM, using Windows XP.

\subsection{Dynamic sensitivity analysis}

Dynamic sensitivities for $G E^{K_{m}}$ parameters behaved similar but in the opposite direction of those for the corresponding $G E^{V_{\max }}$ parameters. The later ones are shown in Figure 3 for the systemic plasma compartment and in Figure 4 for the output compartment. A negative sensitivity means a decrease in $G E^{V_{\max }}$ will result in an increased plasma concentration or amount. The effect on a concentration or amount of a change in a particular $G E^{V_{\max }}$ can be estimated by approximating (1). For instance, a $10 \%$ decrease in $G E_{O A T P}^{V_{\max }}$ with $s_{1, G E_{O A T P}^{V_{\max }}}=-1.2$ at $t=3$ hours (Figure 3, top left), will increase the total paclitaxel concentration in systemic plasma from $\hat{x}_{1}(3)=5.25$ $\mu \mathrm{M}$ (Figure 2, top left) to approximately

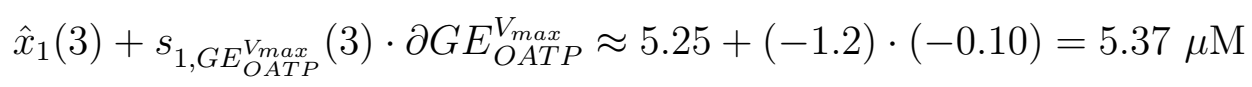

Given the final model estimates, systemic plasma concentration of paclitaxel was clearly most sensitive to changes in $G E_{O A T P}^{V_{\max }}$, describing the uptake by OATP, were a change will have more than 10 times the effect compared to a change in the metabolism by CYP2C 8 at $t=3$ hours, which had the second most sensitive $G E^{V_{\max }}$ (Figure 3, top left). For $6 \alpha$-hydroxypaclitaxel, the ABCB1 transporter is most sensitive, with increasing plasma concentrations for decreasing $G E_{A B C}^{V_{\max }}$, and with CYP2C8 as being second most sensitive, with decreasing plasma concentrations for decreasing $G E_{2 C 8}^{V_{\max }}$ (Figure 3, top right).

In the output compartment, the amount of paclitaxel was most sensitive to changes in $\mathrm{ABCB} 1$, where a decreasing $G E_{A B C}^{V_{\max }}$ will give rise to a decreasing amount (Figure 4, top left). For the amount of $6 \alpha$-hydroxypaclitaxel, metabolism by CYP2C8 is most sensitive, although an equal change in 

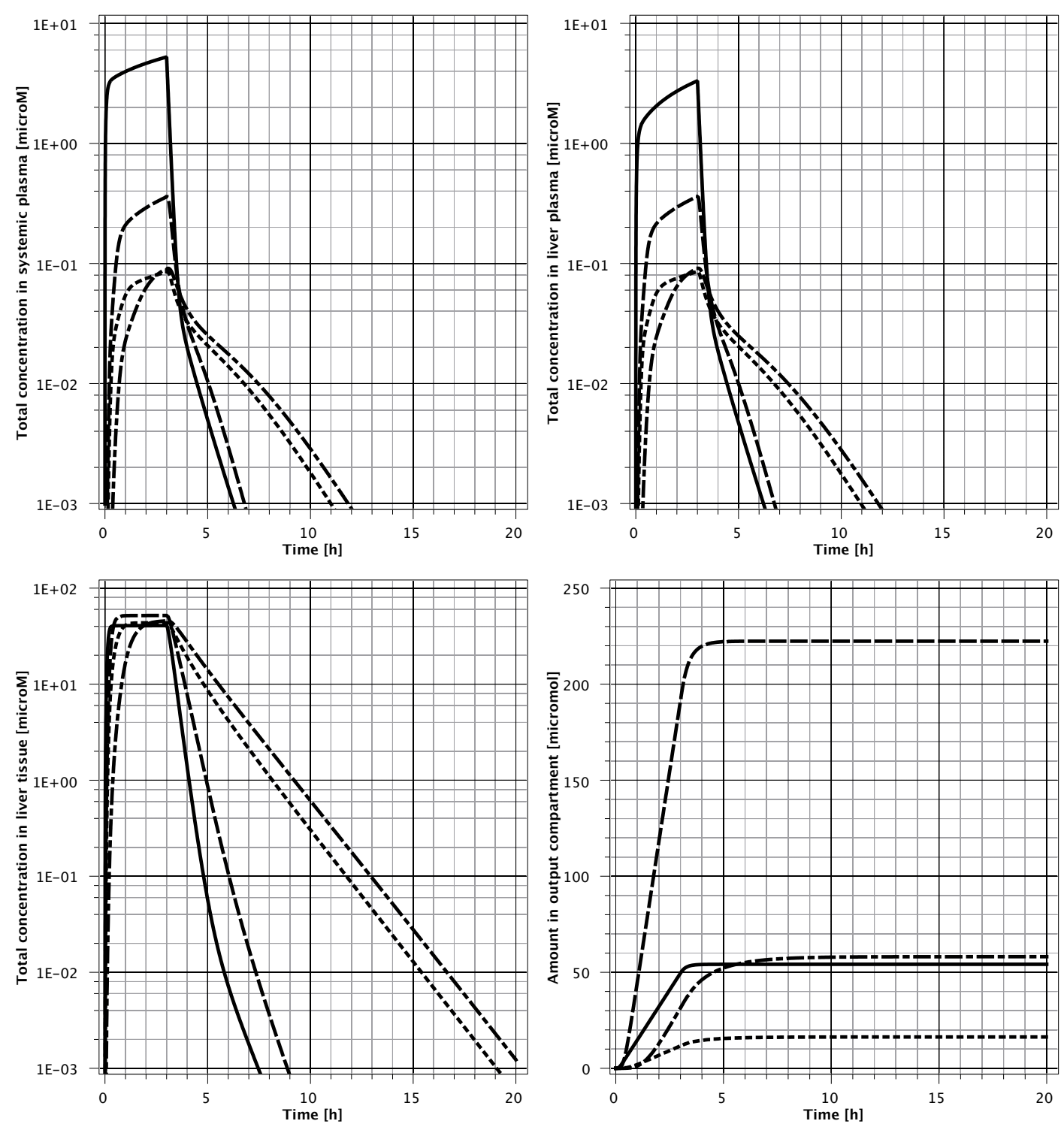

Figure 2: Simulations from the final model of total concentrations in systemic plasma (top left), liver plasma (top right), liver tissue (bottom left) and amounts in output compartment (bottom right) of paclitaxel (solid), 6 $\alpha$-hydroxypaclitaxel (dashed), p-3'-hydroxypaclitaxel (dotted) and 6 $\alpha-$, p-3'-dihydroxypaclitaxel (dash-dotted). 
metabolism by CYP3A4 will have an almost as big but opposite effect (Figure 4, top right). For all compounds in the output compartment, effects from changed uptake by the OATP transporter are small in comparison to changes in metabolism or efflux.

A summary of the effect on paclitaxel and $6 \alpha$-hydroxypaclitaxel of a $10 \%$ decrease in $G E^{V_{\max }}$ can be found in Table 3.

Table 3: Change in concentration and amount from a $10 \%$ decrease in different $G E^{V_{\max }}$

\begin{tabular}{lllll}
\hline Variable $^{a}$ & $G E_{O A T P}^{V_{\max }}=0.9$ & $G E_{2 C 8}^{V_{\max }}=0.9$ & $G E_{3 A 4}^{V_{\max }}=0.9$ & $G E_{A B C}^{V_{\max }}=0.9$ \\
\hline$x_{1}(3)$ & $2.3 \%$ & $0.2 \%$ & $0.1 \%$ & $0.0 \%$ \\
$x_{4}(3)$ & $5.0 \%$ & $-9.4 \%$ & $6.7 \%$ & $34.0 \%$ \\
$x_{13}(18)$ & $0.0 \%$ & $4.1 \%$ & $1.4 \%$ & $-9.0 \%$ \\
$x_{14}(18)$ & $0.0 \%$ & $-3.2 \%$ & $2.3 \%$ & $1.6 \%$ \\
\hline
\end{tabular}

${ }^{a} x_{1}(3)$ : total paclitaxel concentration in systemic plasma at three hours; $x_{4}(3)$ : total $6 \alpha$-hydroxypaclitaxel concentration in systemic plasma at 3 hours; $x_{13}(18)$ : amount paclitaxel in output compartment at 18 hours; $x_{14}(18)$ : amount $6 \alpha$-hydroxypaclitaxel in output compartment at 18 hours

\section{Discussion}

The simulated $C_{\max }$ of $5.25 \mu \mathrm{M}$ for total concentration of paclitaxel at $t=3$ hours in Figure 1 is in the same range as the observed $C_{\max }$ from three-hour infusions reported previously by Walle et al. (1995), with 6-10 $\mu \mathrm{M}$; Karlsson et al. (1999), 2-10 $\mu \mathrm{M}$; Henningsson et al. (2001), 1-10 $\mu \mathrm{M}$; Joerger et al. (2006), 1-4 $\mu \mathrm{M}$. Because of the three-hour constraints in the systemic compartment, maximum total concentrations of metabolites are also in the right range (Czejka et al., 2003; Fransson et al., 2011). The flat phase following the initial rapid increase in concentration and preceding the end of infusion at three hours is not evident from clinical data (Walle et al., 1995; Karlsson et al., 1999; Czejka et al., 2003; Joerger et al., 2006), which could be a consequence of the limited compartmental space in systemic plasma with increased binding as result. Simulation using a ten times larger volume for systemic plasma removed the two distinct phases (data not shown). Previously described population pharmacokinetic models have used two (Henningsson et al., 2001, 2005a; Joerger et al., 2006) or at least one (Henningsson et al., 

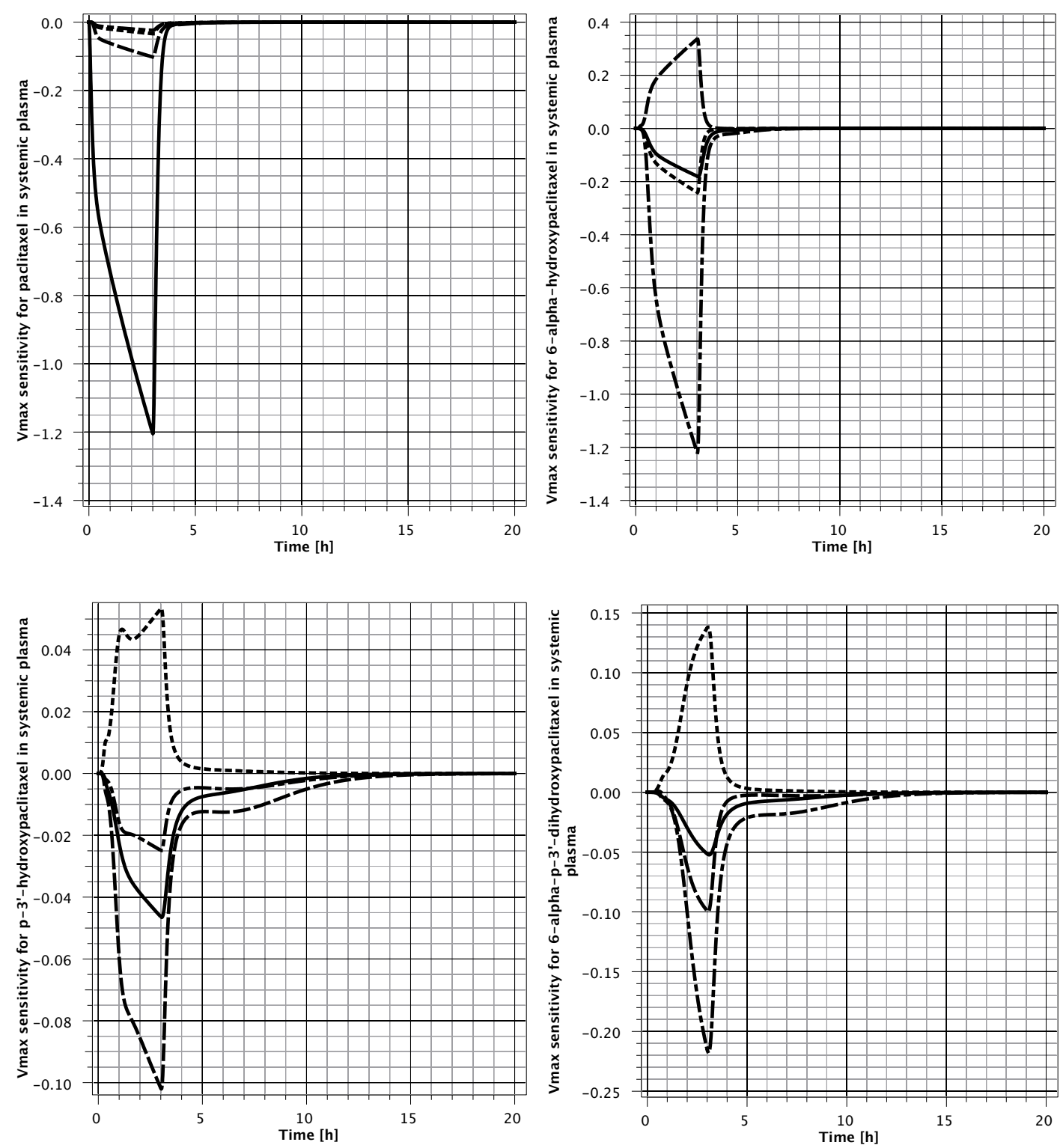

Figure 3: Sensitivity in $G E^{V_{\max }}$ for OATP (solid), CYP2C8 (dashed), CYP3A4 (dotted) and ABCB1 (dash-dotted) in systemic plasma concentrations of paclitaxel (top left), $6 \alpha$-hydroxypaclitaxel (top right), p-3'-hydroxypaclitaxel (bottom left) and 6 $\alpha$-, p-3'-dihydroxypaclitaxel (bottom right). 

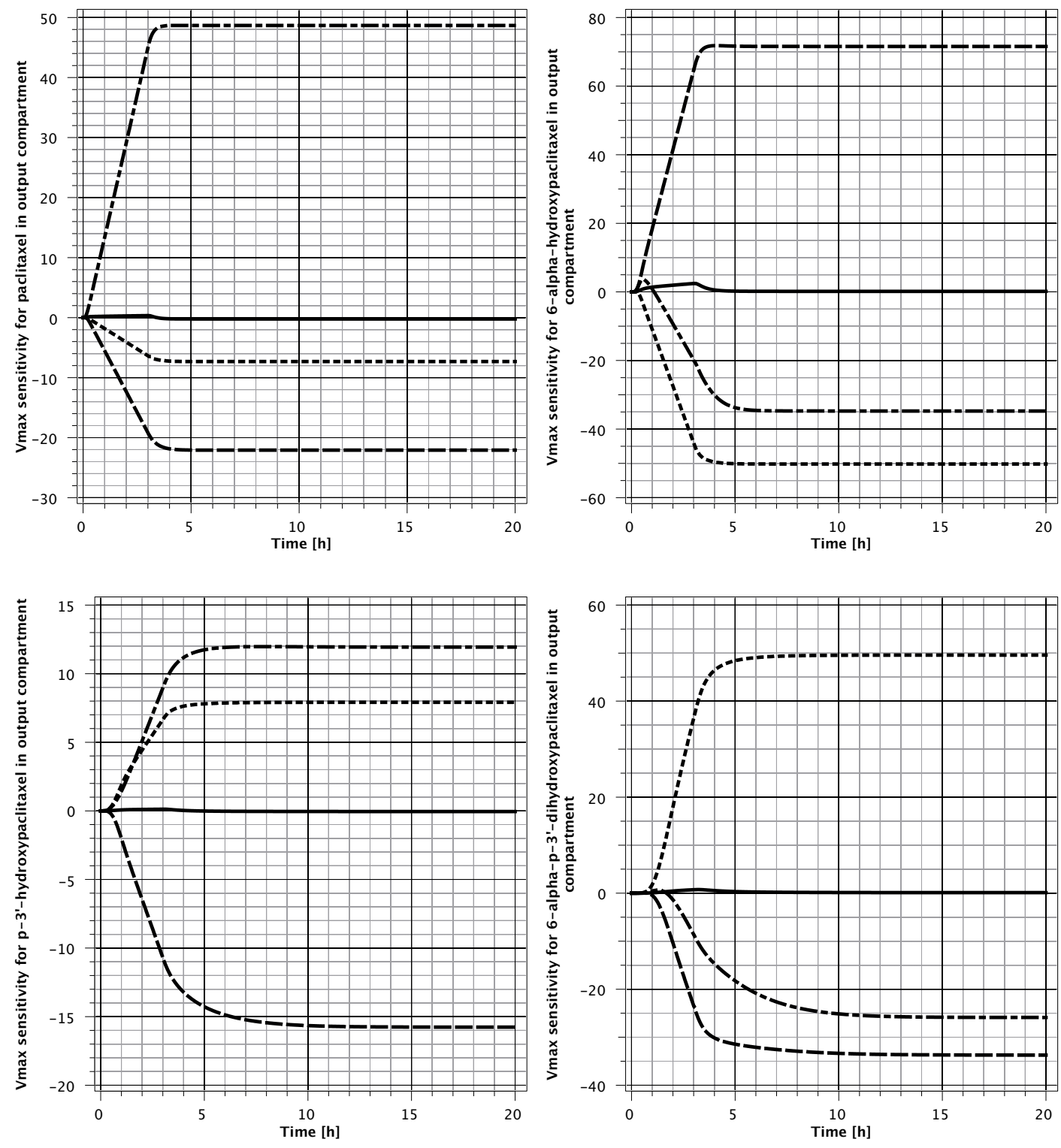

Figure 4: Sensitivity in $G E^{V_{\max }}$ for OATP (solid), CYP2C8 (dashed), CYP3A4 (dotted) and ABCB1 (dash-dotted) in output amounts of paclitaxel (top left), 6 $\alpha$-hydroxypaclitaxel (top right), p-3'-hydroxypaclitaxel (bottom left) and 6 $\alpha^{-}, \mathrm{p}$-3'-dihydroxypaclitaxel (bottom right). 
2003; Bergmann et al., 2011) additional peripheral compartments, although in some cases, for total concentrations of paclitaxel, additional compartments may have been attributable to Cremophor EL binding (Karlsson et al., 1999; Joerger et al., 2006). The lack of peripheral compartments in the present model is also evident from the rapid decrease in total concentrations after the 3 hour infusion. Addition of peripheral compartments to the model were considered, but because previous models are either based on unbound concentrations (Henningsson et al., 2001, 2003, 2005a; Bergmann et al., 2011) or does not explicitly handle binding to Cremophor EL (Karlsson et al., 1999; Joerger et al., 2006), such an approach would mean even more assumptions would need to be accounted for. There are also few sources with parametric models describing the kinetics of paclitaxel metabolites (Fransson et al., 2011).

As a result of the rapid elimination of paclitaxel and metabolites from systemic plasma, amounts in the output compartment are likely to increase and stabilize more rapidly in the present model than in vivo. From Figure 2 (bottom right) it is evident that amounts in the output compartment are stable from approximately 8 hours, which would mean that selecting $t=18$ hours for the constraint on metabolite-drug ratios is sufficient, although the constraint is derived from information on fecal collections between 24-48 hours (Walle et al., 1995). The efflux from liver tissue to the output compartment is fully dependent on the ABCB1 transporter, and no diffusion is assumed with the possibility of a back-flow. A mathematical model by Bartholome et al. (2007) describing vectorial transport by OATP1B3 and ABCC2 across polarized cells found an additional leakage component for efflux over the apical membrane. For the present model, this may indicate that the importance of the transporter ABCB1 will be overestimated for the output compartment. However, because an appropriate volume of the output compartment is difficult to estimate a concentration cannot be determined, something that would be required for a diffusion mechanism. Diffusion would also be affected by the accumulation of the amounts in the output compartment, and this accumulation would not be representative for the in vivo situation.

The total paclitaxel plasma concentration in the systemic compartment is most sensitive to changes in $G E_{O A T P}^{V_{\max }}$. However, the absolute effect on the plasma concentration is small. According to Table 3 a $10 \%$ decrease in $G E_{O A T P}^{V_{\max }}$ will only provide an $2.3 \%$ increase in concentration at the end of infusion at 3 hours. This can be compared to the effect of a $10 \%$ decrease in $G E_{A B C}^{V_{\max }}$, which will cause total concentrations of $6 \alpha$-hydroxypaclitaxel to in- 
crease with $34 \%$ at the same point in time. Given the final model estimates, total paclitaxel concentrations are relatively little affected by changes in GE parameters. If the model and final estimates are to be considered representative for the in vivo situation, it would mean that neither of the investigated transporters, nor the metabolizing enzymes, play a major role in the population variability of paclitaxel plasma concentrations. Hence, they would not show up as significant covariates in clinical studies using population pharmacokinetic data. This may explain the absence of findings by Henningsson et al. (2005a), and the relatively modest effect on decreased clearance by Bergmann et al. (2011). In the same way, the predicted relatively large effect on $6 \alpha$-hydroxypaclitaxel plasma concentrations from decreased capacity in the ABCB1 transporter is supported by our previous findings (Fransson et al., 2011), where individuals carrying the polymorphisms G/A or G/G (wild-type) showed a $30 \%$ increase, and individuals with polymorphism $\mathrm{T} / \mathrm{T}$ showed a $27 \%$ decrease, relative individuals with polymorphism $\mathrm{G} / \mathrm{T}$. The result that genetic variation may influence the metabolite concentrations more than paclitaxel is in accordance with the hypothesis proposed by Leskela et al. (2011). They found that CYP2C8*3, CYP2C8 Haplotype C and CYP3A5*3 correlates to paclitaxel-induced neuropathy and suggested that the metabolites are affecting the risk of neuropathy.

Because local sensitivity analysis is used, the sensitivities in Figures 3 and 4 and the effects in Table 3 are only valid for small deviations from the final parameter estimates (Appendix, Table A.5). The validity of the sensitivity analysis can be tested by manually reducing the parameter estimates and then perform a new simulation. Such simulations showed that a $10 \%$ decrease in the estimate agree with the local sensitivity analysis, but that a $50 \%$ decrease in some cases are too big, and that the sensitivity plots in Figures 3 and 4 cannot be used to accurately predict such a large deviation (data not shown). This should be considered if effects from genetic polymorphisms are considered to be large, in which case the solution would be an additional simulation using a reduced estimate.

In conclusion, the developed model predicts plasma concentrations of drug and metabolites that are in the range of observations from clinical studies. Given the final model structure with parameter estimates derived from constrained optimization, while plasma concentrations of paclitaxel seems to be relatively little affected by changes in the capacity of transport or metabolism, its main metabolite $6 \alpha$-hydroxypaclitaxel may be largely affected even by small changes. If future studies can confirm that paclitaxel 
metabolites are clinically relevant, the present work indicates that genetic polymorphisms may play an important role for individualizing paclitaxel treatment.

\section{Acknowledgements}

This work has been supported by the Swedish Knowledge Foundation through the Industrial $\mathrm{PhD}$ programme in Medical Bioinformatics at the Strategy and Development Office (SDO) at Karolinska Institutet, the Swedish Cancer Society and the Swedish Research Council.

\section{References}

Aarons, L., 2005. Physiologically based pharmacokinetic modelling: a sound mechanistic basis is needed. Br J Clin Pharmacol 60, 581-583.

Barter, Z.E., Bayliss, M.K., Beaune, P.H., Boobis, A.R., Carlile, D.J., Edwards, R.J., Houston, J.B., Lake, B.G., Lipscomb, J.C., Pelkonen, O.R., Tucker, G.T., Rostami-Hodjegan, A., 2007. Scaling factors for the extrapolation of in vivo metabolic drug clearance from in vitro data: reaching a consensus on values of human microsomal protein and hepatocellularity per gram of liver. Curr. Drug Metab. 8, 33-45.

Bartholome, K., Rius, M., Letschert, K., Keller, D., Timmer, J., Keppler, D., 2007. Data-based mathematical modeling of vectorial transport across double-transfected polarized cells. Drug Metab. Dispos. 35, 1476-1481.

Bergmann, T.K., Brasch-Andersen, C., Green, H., Mirza, M., Pedersen, R.S., Nielsen, F., Skougaard, K., Wihl, J., Keldsen, N., Damkier, P., Friberg, L.E., Peterson, C., Vach, W., Karlsson, M.O., Brosen, K., 2011. Impact of CYP2C8*3 on paclitaxel clearance: a population pharmacokinetic and pharmacogenomic study in 93 patients with ovarian cancer. Pharmacogenomics J. 11, 113-120.

Cresteil, T., Monsarrat, B., Alvinerie, P., Treluyer, J.M., Vieira, I., Wright, M., 1994. Taxol metabolism by human liver microsomes: identification of cytochrome P450 isozymes involved in its biotransformation. Cancer Res. 54, 386-392. 
Cresteil, T., Monsarrat, B., Dubois, J., Sonnier, M., Alvinerie, P., Gueritte, F., 2002. Regioselective metabolism of taxoids by human CYP3A4 and 2C8: structure-activity relationship. Drug Metab. Dispos. 30, 438-445.

Czejka, M., Schueller, J., Schnait, H., Springer, B., Eder, I., 2003. Clinical pharmacokinetics and metabolism of paclitaxel after a three-hour infusion: comparison of two preparations. J Oncol Pharm Pract 9, 129-136.

Fransson, M.N., Green, H., Litton, J.E., Friberg, L.E., 2011. Influence of Cremophor EL and genetic polymorphisms on the pharmacokinetics of paclitaxel and its metabolites using a mechanism-based model. Drug Metab. Dispos. 39, 247-255.

Geraghty, E.M., Boone, J.M., McGahan, J.P., Jain, K., 2004. Normal organ volume assessment from abdominal CT. Abdom Imaging 29, 482-490.

de la Grandmaison, G.L., Clairand, I., Durigon, M., 2001. Organ weight in 684 adult autopsies: new tables for a Caucasoid population. Forensic Sci. Int. 119, 149-154.

Green, H., Soderkvist, P., Rosenberg, P., Mirghani, R.A., Rymark, P., Lundqvist, E.A., Peterson, C., 2009. Pharmacogenetic studies of Paclitaxel in the treatment of ovarian cancer. Basic Clin. Pharmacol. Toxicol. 104, 130-137.

Harris, J.W., Katki, A., Anderson, L.W., Chmurny, G.N., Paukstelis, J.V., Collins, J.M., 1994a. Isolation, structural determination, and biological activity of 6 alpha-hydroxytaxol, the principal human metabolite of taxol. J. Med. Chem. 37, 706-709.

Harris, J.W., Rahman, A., Kim, B.R., Guengerich, F.P., Collins, J.M., $1994 b$. Metabolism of taxol by human hepatic microsomes and liver slices: participation of cytochrome P450 3A4 and an unknown P450 enzyme. Cancer Res. 54, 4026-4035.

Henningsson, A., Karlsson, M.O., Vigano, L., Gianni, L., Verweij, J., Sparreboom, A., 2001. Mechanism-based pharmacokinetic model for paclitaxel. J. Clin. Oncol. 19, 4065-4073.

Henningsson, A., Marsh, S., Loos, W.J., Karlsson, M.O., Garsa, A., Mross, K., Mielke, S., Vigano, L., Locatelli, A., Verweij, J., Sparreboom, A., 
McLeod, H.L., 2005a. Association of CYP2C8, CYP3A4, CYP3A5, and ABCB1 polymorphisms with the pharmacokinetics of paclitaxel. Clin. Cancer Res. 11, 8097-8104.

Henningsson, A., Sparreboom, A., Loos, W.J., Verweij, J., Silvander, M., Karlsson, M.O., 2005b. Population pharmacokinetic model for Cremophor EL (Abstract). PAGE 14, 770.

Henningsson, A., Sparreboom, A., Sandstrom, M., Freijs, A., Larsson, R., Bergh, J., Nygren, P., Karlsson, M.O., 2003. Population pharmacokinetic modelling of unbound and total plasma concentrations of paclitaxel in cancer patients. Eur. J. Cancer 39, 1105-1114.

Hindmarsh, A.C., Brown, P.N., Grant, K.E., Lee, S.L., Serban, R., Shumaker, D.E., Woodward, C.S., 2005. SUNDIALS: Suite of nonlinear and differential/algebraic equation solvers. ACM Transactions on Mathematical Software 31, 363-396.

Hurley, P.J., 1975. Red cell and plasma volumes in normal adults. J. Nucl. Med. 16, 46-52.

Jang, S.H., Wientjes, M.G., Au, J.L., 2001. Kinetics of P-glycoproteinmediated efflux of paclitaxel. J. Pharmacol. Exp. Ther. 298, 1236-1242.

Joerger, M., Huitema, A.D., van den Bongard, D.H., Schellens, J.H., Beijnen, J.H., 2006. Quantitative effect of gender, age, liver function, and body size on the population pharmacokinetics of Paclitaxel in patients with solid tumors. Clin. Cancer Res. 12, 2150-2157.

Karlsson, M.O., Molnar, V., Freijs, A., Nygren, P., Bergh, J., Larsson, R., 1999. Pharmacokinetic models for the saturable distribution of paclitaxel. Drug Metab. Dispos. 27, 1220-1223.

Kato, M., Chiba, K., Ito, T., Koue, T., Sugiyama, Y., 2010. Prediction of interindividual variability in pharmacokinetics for CYP3A4 substrates in humans. Drug Metab. Pharmacokinet. 25, 367-378.

Kuh, H.J., Jang, S.H., Wientjes, M.G., Au, J.L., 2000. Computational model of intracellular pharmacokinetics of paclitaxel. J. Pharmacol. Exp. Ther. 293, 761-770. 
Kumar, G.N., Oatis, J.E., Thornburg, K.R., Heldrich, F.J., Hazard, E.S., Walle, T., 1994. 6 alpha-Hydroxytaxol: isolation and identification of the major metabolite of taxol in human liver microsomes. Drug Metab. Dispos. $22,177-179$.

Leskela, S., Jara, C., Leandro-Garcia, L.J., Martinez, A., Garcia-Donas, J., Hernando, S., Hurtado, A., Vicario, J.C., Montero-Conde, C., Landa, I., Lopez-Jimenez, E., Cascon, A., Milne, R.L., Robledo, M., RodriguezAntona, C., 2011. Polymorphisms in cytochromes P450 2C8 and 3A5 are associated with paclitaxel neurotoxicity. Pharmacogenomics J. 11, 121129.

Molino, G., Avagnina, P., Ballare, M., Torchio, M., Niro, A.G., Aurucci, P.E., Grosso, M., Fava, C., 1991. Combined evaluation of total and functional liver plasma flows and intrahepatic shunting. Dig. Dis. Sci. 36, 1189-1196.

Monsarrat, B., Alvinerie, P., Wright, M., Dubois, J., Gueritte-Voegelein, F., Guenard, D., Donehower, R.C., Rowinsky, E.K., 1993. Hepatic metabolism and biliary excretion of Taxol in rats and humans. J. Natl. Cancer Inst. Monographs , 39-46.

Nakajima, M., Fujiki, Y., Kyo, S., Kanaya, T., Nakamura, M., Maida, Y., Tanaka, M., Inoue, M., Yokoi, T., 2005. Pharmacokinetics of paclitaxel in ovarian cancer patients and genetic polymorphisms of CYP2C8, CYP3A4, and MDR1. J Clin Pharmacol 45, 674-682.

Naraharisetti, S.B., Lin, Y.S., Rieder, M.J., Marciante, K.D., Psaty, B.M., Thummel, K.E., Totah, R.A., 2010. Human liver expression of CYP2C8: gender, age, and genotype effects. Drug Metab. Dispos. 38, 889-893.

Rahman, A., Korzekwa, K.R., Grogan, J., Gonzalez, F.J., Harris, J.W., 1994. Selective biotransformation of taxol to 6 alpha-hydroxytaxol by human cytochrome P450 2C8. Cancer Res. 54, 5543-5546.

Sirianni, G.L., Pang, K.S., 1997. Organ clearance concepts: new perspectives on old principles. J Pharmacokinet Biopharm 25, 449-470.

Smith, N.F., Acharya, M.R., Desai, N., Figg, W.D., Sparreboom, A., 2005. Identification of OATP1B3 as a high-affinity hepatocellular transporter of paclitaxel. Cancer Biol. Ther. 4, 815-818. 
Sonnichsen, D.S., Liu, Q., Schuetz, E.G., Schuetz, J.D., Pappo, A., Relling, M.V., 1995. Variability in human cytochrome P450 paclitaxel metabolism. J. Pharmacol. Exp. Ther. 275, 566-575.

Sparreboom, A., Figg, W.D., 2006. Identifying sources of interindividual pharmacokinetic variability with population modeling. Clin. Cancer Res. 12, 1951-1953.

Sparreboom, A., van Tellingen, O., Nooijen, W.J., Beijnen, J.H., 1996. Nonlinear pharmacokinetics of paclitaxel in mice results from the pharmaceutical vehicle Cremophor EL. Cancer Res. 56, 2112-2115.

Taniguchi, H., Masuyama, M., Koyama, H., Oguro, A., Takahashi, T., 1996. Quantitative measurement of human tissue hepatic blood volume by $\mathrm{C} 15 \mathrm{O}$ inhalation with positron-emission tomography. Liver 16, 258-262.

Undevia, S.D., Gomez-Abuin, G., Ratain, M.J., 2005. Pharmacokinetic variability of anticancer agents. Nat. Rev. Cancer 5, 447-458.

Varma, A., Morbidelli, M., Wu, H., 1999. Parametric sensitivity in chemical systems. Cambridge series in chemical engineering, Cambridge University Press.

Walle, T., Walle, U.K., Kumar, G.N., Bhalla, K.N., 1995. Taxol metabolism and disposition in cancer patients. Drug Metab. Dispos. 23, 506-512.

Wu, W.H., Wang, F.S., Chang, M.S., 2008. Dynamic sensitivity analysis of biological systems. BMC Bioinformatics 9 Suppl 12, S17.

Yamaguchi, H., Hishinuma, T., Endo, N., Tsukamoto, H., Kishikawa, Y., Sato, M., Murai, Y., Hiratsuka, M., Ito, K., Okamura, C., Yaegashi, N., Suzuki, N., Tomioka, Y., Goto, J., 2006. Genetic variation in ABCB1 influences paclitaxel pharmacokinetics in Japanese patients with ovarian cancer. Int. J. Gynecol. Cancer 16, 979-985. 


\section{Appendix A. Model equations and parameter estimates}

$$
\begin{aligned}
& V_{S y s P l} \cdot \dot{x}_{1}=-Q_{L i v P l}\left(x_{1}-x_{2}\right)+D_{o s e}^{p a c} \\
& x_{1}=y_{1}+\left(B_{l i n P l}+B_{C r E L}^{p a c} \cdot x_{17}\right) \cdot y_{1}+\frac{B_{\operatorname{maxPl}} \cdot y_{1}}{K_{m P l}+y_{1}} \\
& V_{L i v P l} \cdot \dot{x}_{2}=Q_{L i v P l}\left(x_{1}-x_{2}\right)-Q_{L i v T i}\left(y_{2}-y_{3}\right)-\frac{V_{m a x O A T P}^{p a c} \cdot y_{2}}{K_{m O A T P}^{p a c}+y_{2}} \\
& x_{2}=y_{2}+\left(B_{l i n P l}+B_{C r E L}^{p a c} \cdot x_{17}\right) \cdot y_{2}+\frac{B_{\max P l} \cdot y_{2}}{K_{m P l}+y_{2}} \\
& V_{L i v T i} \cdot \dot{x}_{3}=Q_{L i v T i}\left(y_{2}-y_{3}\right)+\frac{V_{\text {maxOATP }}^{\text {pac }} \cdot y_{2}}{K_{m O A T P}^{\text {pac }}+y_{2}} \\
& -\frac{V_{m a x 2 C 8}^{p a c} \cdot y_{3}}{K_{m 2 C 8}^{p a c}+y_{3}}-\frac{V_{m a x}^{p a A 4} \cdot y_{3}}{K_{m 3 A 4}^{p a c}+y_{3}}-\frac{V_{m a x}^{p a c}}{K_{m A B C}^{p a c}+y_{3}} \\
& x_{3}=y_{3}+B_{\text {linTi }} \cdot y_{3}+\frac{B_{\max T i} \cdot y_{3}}{K_{m T i}+y_{3}} \\
& \dot{x}_{13}=\frac{V_{\max A B C}^{\text {pac }} \cdot y_{3}}{K_{m A B C}^{\text {pac }}+y_{3}} \\
& V_{S y s P l} \cdot \dot{x}_{4}=-Q_{L i v P l}\left(x_{4}-x_{5}\right) \\
& x_{4}=y_{4}+\left(B_{l i n P l}+B_{C r E L}^{6 \alpha} \cdot x_{17}\right) \cdot y_{4}+\frac{B_{\operatorname{maxPl}} \cdot y_{4}}{K_{m P l}+y_{4}} \\
& V_{L i v P l} \cdot \dot{x}_{5}=Q_{L i v P l}\left(x_{4}-x_{5}\right)-Q_{L i v T i}\left(y_{5}-y_{6}\right)-\frac{V_{\text {maxOATP }}^{6 \alpha} \cdot y_{5}}{K_{m O A T P}^{6 \alpha}+y_{5}} \\
& x_{5}=y_{5}+\left(B_{l i n P l}+B_{C r E L}^{6 \alpha} \cdot x_{17}\right) \cdot y_{5}+\frac{B_{\operatorname{maxPl}} \cdot y_{5}}{K_{m P l}+y_{5}} \\
& V_{L i v T i} \cdot \dot{x}_{6}=Q_{L i v T i}\left(y_{5}-y_{6}\right)+\frac{V_{\operatorname{maxOATP}}^{6 \alpha} \cdot y_{5}}{K_{m O A T P}^{6 \alpha}+y_{5}} \\
& +\frac{V_{m a x 2 C 8}^{p a c} \cdot y_{3}}{K_{m 2 C 8}^{p a c}+y_{3}}-\frac{V_{m a x 3 A 4}^{6 \alpha} \cdot y_{6}}{K_{m 3 A 4}^{6 \alpha}+y_{6}}-\frac{V_{m a x A B C}^{6 \alpha} \cdot y_{6}}{K_{m A B C}^{6 \alpha}+y_{6}} \\
& x_{6}=y_{6}+B_{l i n T i} \cdot y_{6}+\frac{B_{m a x T i} \cdot y_{6}}{K_{m T i}+y_{6}} \\
& \dot{x}_{14}=\frac{V_{\operatorname{maxABC}}^{6 \alpha} \cdot y_{6}}{K_{m A B C}^{6 \alpha}+y_{6}}
\end{aligned}
$$




$$
\begin{aligned}
& V_{S y s P l} \cdot \dot{x}_{7}=-Q_{L i v P l}\left(x_{7}-x_{8}\right) \\
& x_{7}=y_{7}+\left(B_{l i n P l}+B_{C r E L}^{p-3^{\prime}} \cdot x_{17}\right) \cdot y_{7}+\frac{B_{\operatorname{maxPl}} \cdot y_{7}}{K_{m P l}+y_{7}} \\
& V_{L i v P l} \cdot \dot{x}_{8}=Q_{L i v P l}\left(x_{7}-x_{8}\right)-Q_{L i v T i}\left(y_{8}-y_{9}\right)-\frac{V_{m a x O A T P}^{p-3^{\prime}} \cdot y_{8}}{K_{m O A T P}^{p-3^{\prime}}+y_{8}} \\
& x_{8}=y_{8}+\left(B_{l i n P l}+B_{C r E L}^{p-3^{\prime}} \cdot x_{17}\right) \cdot y_{8}+\frac{B_{\max P l} \cdot y_{8}}{K_{m P l}+y_{8}} \\
& V_{L i v T i} \cdot \dot{x}_{9}=Q_{L i v T i}\left(y_{8}-y_{9}\right)+\frac{V_{\operatorname{maxOATP}}^{p-3^{\prime}} \cdot y_{8}}{K_{m O A T P}^{p-3^{\prime}}+y_{8}} \\
& +\frac{V_{\max 3 A 4}^{p a c} \cdot y_{3}}{K_{m 3 A 4}^{p a c}+y_{3}}-\frac{V_{\max 2 C 8}^{p-3^{\prime}} \cdot y_{9}}{K_{m 2 C 8}^{p-3^{\prime}}+y_{9}}-\frac{V_{\operatorname{maxABC}}^{p-3^{\prime}} \cdot y_{9}}{K_{m A B C}^{p-3^{\prime}}+y_{9}} \\
& x_{9}=y_{9}+B_{l i n T i} \cdot y_{9}+\frac{B_{\max T i} \cdot y_{9}}{K_{m T i}+y_{9}} \\
& \dot{x}_{15}=\frac{V_{\operatorname{maxABC}}^{p-3^{\prime}} \cdot y_{9}}{K_{m A B C}^{p-3^{\prime}}+y_{9}}
\end{aligned}
$$




$$
\begin{aligned}
& V_{S y s P l} \cdot \dot{x}_{10}=-Q_{\text {LivPl }}\left(x_{10}-x_{11}\right) \\
& x_{10}=y_{10}+\left(B_{l i n P l}+B_{C r E L}^{d i} \cdot x_{17}\right) \cdot y_{10}+\frac{B_{\max P l} \cdot y_{10}}{K_{m P l}+y_{10}} \\
& V_{L i v P l} \cdot \dot{x}_{11}=Q_{L i v P l}\left(x_{10}-x_{11}\right)-Q_{L i v T i}\left(y_{11}-y_{12}\right)-\frac{V_{m a x O A T P}^{d i} \cdot y_{11}}{K_{m O A T P}^{d i}+y_{11}} \\
& x_{11}=y_{11}+\left(B_{l i n P l}+B_{C r E L}^{d i} \cdot x_{17}\right) \cdot y_{11}+\frac{B_{\max P l} \cdot y_{11}}{K_{m P l}+y_{11}} \\
& V_{L i v T i} \cdot \dot{x}_{12}=Q_{L i v T i}\left(y_{11}-y_{12}\right)+\frac{V_{m a x O A T P}^{d i} \cdot y_{11}}{K_{m O A T P}^{d i}+y_{11}} \\
& +\frac{V_{m a x 3 A 4}^{6 \alpha} \cdot y_{6}}{K_{m 3 A 4}^{6 \alpha}+y_{6}}+\frac{V_{m a x 2 C 8}^{p-3^{\prime}} \cdot y_{9}}{K_{m 2 C 8}^{p-3^{\prime}}+y_{9}}-\frac{V_{\operatorname{maxABC}}^{d i} \cdot y_{12}}{K_{m A B C}^{d i}+y_{12}} \\
& x_{12}=y_{12}+B_{l i n T i} \cdot y_{12}+\frac{B_{\max T i} \cdot y_{12}}{K_{m T i}+y_{12}} \\
& \dot{x}_{16}=\frac{V_{\operatorname{maxABC}}^{d i} \cdot y_{12}}{K_{m A B C}^{d i}+y_{12}} \\
& V_{1}^{C r E L} \cdot \dot{x}_{17}=-Q_{12}^{C r E L}\left(x_{17}-x_{18}\right)-Q_{13}^{C r E L}\left(x_{17}-x_{19}\right) \\
& -\frac{V_{\max }^{C r E L} \cdot x_{17}}{K_{m}^{C r E L}+x_{17}}+D_{o s e}^{C r E L} \\
& V_{2}^{C r E L} \cdot \dot{x}_{18}=Q_{12}^{C r E L}\left(x_{17}-x_{18}\right) \\
& V_{3}^{C r E L} \cdot \dot{x}_{19}=Q_{13}^{C r E L}\left(x_{17}-x_{19}\right)
\end{aligned}
$$




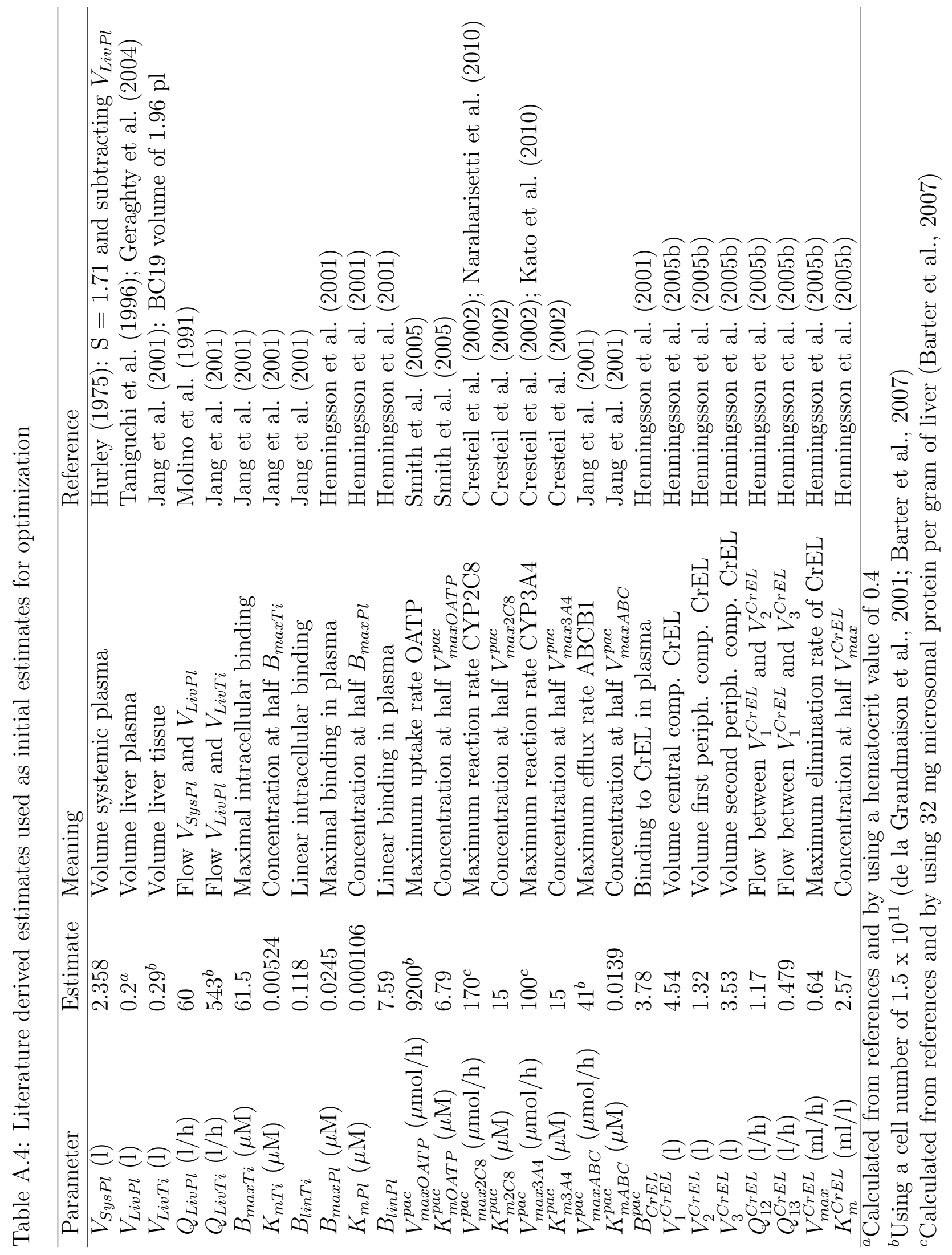


Table A.5: Estimates from constrained optimization for paclitaxel transport, metabolism and Cremophor EL binding

\begin{tabular}{|c|c|c|c|}
\hline Parameter & Initial $^{a}$ & Manual $^{b}$ & Mathematica $^{c}$ \\
\hline$V_{\text {maxOATP }}^{\text {pac }}(\mu \mathrm{mol} / \mathrm{h})$ & 9200 & 2300 & 2210 \\
\hline$K_{m O A T P}^{\text {pac }}(\mu \mathrm{M})$ & 6.79 & fixed & fixed \\
\hline$V_{\max 2 C 8}^{\text {pac }}(\mu \mathrm{mol} / \mathrm{h})$ & 170 & 68000 & 108000 \\
\hline$K_{m 2 C 8}^{p a c}(\mu \mathrm{M})$ & 15 & fixed & fixed \\
\hline$V_{\max 3 A 4}^{\text {pac }}(\mu \mathrm{mol} / \mathrm{h})$ & 100 & 40000 & 35800 \\
\hline$K_{m 3 A 4}^{p a c}(\mu \mathrm{M})$ & 15 & fixed & fixed \\
\hline$V_{\max A B C}^{\text {pac }}(\mu \mathrm{mol} / \mathrm{h})$ & 41 & fixed & fixed \\
\hline$K_{m A B C}^{\text {pac }}(\mu \mathrm{M})$ & 0.0139 & fixed & fixed \\
\hline$V_{\max O A T P}^{6 \alpha}(\mu \mathrm{mol} / \mathrm{h})$ & 9200 & 4600 & 4030 \\
\hline$K_{m O A T P}^{6 \alpha}(\mu \mathrm{M})$ & 6.79 & fixed & 5.79 \\
\hline$V_{\max 3 A 4}^{6 \alpha}(\mu \mathrm{mol} / \mathrm{h})$ & 100 & 100 & 114 \\
\hline$K_{m 3 A 4}^{6 \alpha}(\mu \mathrm{M})$ & 15 & fixed & 16.3 \\
\hline$V_{\max A B C}^{6 \alpha}(\mu \mathrm{mol} / \mathrm{h})$ & 41 & 82 & 107 \\
\hline$K_{m A B C}^{6 \alpha}(\mu \mathrm{M})$ & 0.0139 & fixed & 0.0130 \\
\hline$V_{\max O A T P}^{p-3^{\prime}}(\mu \mathrm{mol} / \mathrm{h})$ & 9200 & 18400 & 14000 \\
\hline$K_{m O A T P}^{p-3^{\prime}}(\mu \mathrm{M})$ & 6.79 & fixed & 8.43 \\
\hline$V_{\max 2 C 8}^{p-3^{\prime}}(\mu \mathrm{mol} / \mathrm{h})$ & 170 & 17000 & 16900 \\
\hline$K_{m 2 C 8}^{p-3^{\prime}}(\mu \mathrm{M})$ & 15 & fixed & 11.0 \\
\hline$V_{\max A B C}^{p-3^{\prime}}(\mu \mathrm{mol} / \mathrm{h})$ & 41 & 10 & 10.4 \\
\hline$K_{m A B C}^{p-3^{\prime}}(\mu \mathrm{M})$ & 0.0139 & fixed & 0.0143 \\
\hline$V_{\max }^{\text {di }}{ }^{2}(\mu \mathrm{mol} / \mathrm{h})$ & 9200 & 18400 & 15200 \\
\hline$K_{m O A T P}^{d i}(\mu \mathrm{M})$ & 6.79 & fixed & 7.37 \\
\hline$V_{\max A B C}^{d i}(\mu \mathrm{mol} / \mathrm{h})$ & 41 & 41 & 34.8 \\
\hline$K_{m A B C}^{d i}(\mu \mathrm{M})$ & 0.0139 & fixed & 0.0119 \\
\hline$B_{C r E L}^{p a c}$ & 3.78 & fixed & fixed \\
\hline$B_{C r E L}^{6 \alpha}$ & 3.78 & fixed & 4.79 \\
\hline$B_{C r E L}^{p-3^{\prime}}$ & 3.78 & fixed & 2.85 \\
\hline$B_{C r E L}^{d i}$ & 3.78 & fixed & 3.34 \\
\hline
\end{tabular}

${ }^{a}$ From Table A.4. Estimates for parameters governing metabolite kinetics are assumed to be the same as for the parent drug.

${ }^{b}$ Using MathModelica Simulation Center by adjusting $V_{\max }$ parameters to meet an approximate $50 \%$ deviation from the constraints.

${ }^{c}$ Final estimates. Using Mathematica with NDSolve and FindMinimum to meet a maximum of $20 \%$ deviation from the constraints. 